\title{
Effect of grafting on the binding transition of two flexible polymers
}

\author{
Johannes Zierenberg ${ }^{\mathrm{a}}$, Katharina Tholen ${ }^{\mathrm{b}}$, and Wolfhard Janke ${ }^{\mathrm{c}}$ \\ Institut für Theoretische Physik, Universität Leipzig, Postfach 100 920, D-04009 Leipzig, \\ Germany
}

\begin{abstract}
We investigate the binding transition of two flexible polymers grafted to a steric surface with closeby end points. While free polymers show a discontinuous transition, grafting to a steric flat surface leads to a continuous binding transition. This is supported by results from Metropolis and parallel multicanonical simulations. A combination of canonical and microcanonical analyses reveals that the change in transition order can be understood in terms of the reduced translational entropy of the unbound high-temperature phase upon grafting.
\end{abstract}

\section{Introduction}

To study physical or chemical properties of polymers, one typically needs to locally fix them. In the context of semiflexible polymer bundles, one can experimentally rely on a multitude of methods such as fluorescence microscopy, optical tweezers, atomic force microscopy, light scattering, electron microscopy, and X-ray diffraction [1]. For example, semiflexible biopolymer bundles may be attached to polystyrene beads for a contact-free manipulation via optical tweezers. Actin filaments are a good candidate to study bundles of semiflexible polymers and their response to physical forces [2]3. The stiffening of actin bundles can be adjusted by cross-linking proteins 4/5]. Due to the stiff nature of biopolymers, this can be well-described using the worm-like chain model [6]. This further allows one to study the binding mechanism of directed polymers [78] or polymers under tension [9], as well as force-induced desorption or unzipping [10.

Introducing attractive interactions among all monomers along the chain including self-avoidance leads to so-called self-attractive semiflexible (theta) polymers [1]. This formulation allows studies of the competition between collapse and bundling, e.g., in the context of DNA molecules [12] where, depending on DNA and condensing agent concentration, bundles of finite thickness were observed both numerically and experimentally. In general, it was observed that bundles of semiflexible polymers form twisted structures, which may be attributed to interaction-surface maximization 6 13 14 15. The tendency to form polymer bundles requires sufficient (effective)

\footnotetext{
a e-mail: johannes.zierenberg@itp.uni-leipzig.de

b e-mail: katharina.tholen@itp.uni-leipzig.de

c e-mail: wolfhard.janke@itp.uni-leipzig.de
} 
stiffness; for flexible polymers both static and dynamic reasons prevent bundle formation 1516 .

For the study of single or isolated polymer behavior, one often grafts the chains to substrates (see, e.g., Refs. 17 18]). Depending on the setup, an interaction with neighboring chains cannot be completely excluded. In contrast, increasing the density of grafted polymer systems, semiflexible (theta) polymers were shown to exhibit a rich phase space forming toroidal, archway and tower micelles 192021 for which they need to locally form bundles and which depends on both chain length and density. A recent approach to systematically study the competition between long-range charge repulsion and short-range attraction involves grafted polymers that are allowed to move freely on the surface [22, finding finite-size bundles as well as formation of infinite bundles depending on the range of repulsive interactions.

Here, we study the binding of two flexible polymers grafted closeby to a steric surface. This is in contrast to the directed or semiflexible approaches to study binding and allows one to compare to the uncorrelated motifs that originate within aggregation of flexible polymers 15 . We notice that there has been a somewhat equivalent use of the terms aggregation 232425 and binding 78 . Here, we refer to binding as the process of two polymers attaching to each other, in our case even flexible polymers. If specific inter-polymer interactions are considered this may quickly lead to effects also characterized as zipping (see, e.g., Refs. 26 27/28|29|30|31]). Even more interesting is the equivalence between two-polymer binding of directed polymers and adsorption [7. This should qualitatively remain valid also for flexible polymers [32, especially if one imagines the crossover scenario of a flexible polymer adsorbed to a nanowire, equivalent to the stiff limit of a polymer chain 3334, or a flexible polymer adsorbing to a flexible surface 35 . In fact, it was shown that grafting alters the firstorder-like adsorption transition to a second-order-like transition 36. We will show below that the analogous scenario holds true for the binding of two flexible polymers, where close-by-grafting may lead to single-polymer behavior.

The rest of the paper is organized as follows: After introducing the investigated model and employed methods in Sec. 2, we present our results in the canonical (Sec. 3.1) and microcanonical (Sec. 3.2) ensemble. We finish with our conclusions in Sec. 4

\section{Model and Methods}

A generic coarse-grained polymer model is a linear bead-spring homopolymer. In principle, this allows one to model systems with linear chains from simple synthetic polymers such as polyethylene to biological biopolymers such as DNA or actin filaments if stiffness is included. In our case, monomers are connected by the finitely extensible nonlinear elastic (FENE) potential,

$$
V_{\mathrm{FENE}}(r)=-\frac{K}{2} R^{2} \ln \left(1-\left[\left(r-r_{0}\right) / R\right]^{2}\right),
$$

acting between bonded monomers. This is locally harmonic but diverges for $\mid r-$ $r_{0} \mid \rightarrow R$, where $r_{0}=0.7$ is the "equilibrium" bond length (if no other potential was involved), $R=0.3$ and $K=40$. Non-bonded monomers interact via the LennardJones potential,

$$
V_{\mathrm{LJ}}(r)=4 \epsilon\left[(\sigma / r)^{12}-(\sigma / r)^{6}\right]=\epsilon\left[\left(r_{0} / r\right)^{12}-2\left(r_{0} / r\right)^{6}\right],
$$

where $\sigma=2^{-1 / 6} r_{0}$ connects the non-bonded length scale to the equilibrium bond length and $\epsilon=1$ sets the energy scale. The repulsive $r^{-12}$ term models self-avoidance, 

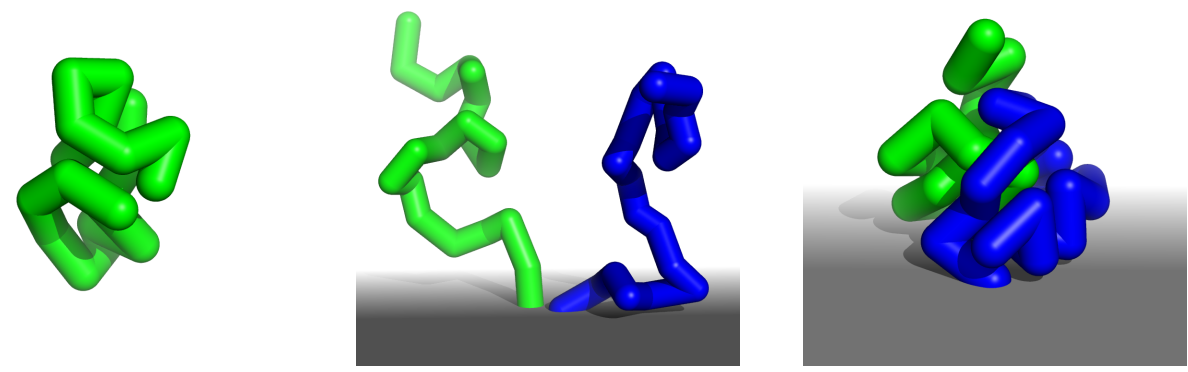

Fig. 1. Snapshots of a single polymer of length $N=20$ in the globular phase below the collapse transition (left, $T=0.7$ ) and two polymers of length $N=20$ grafted to a steric surface above (middle, $T=4$ ) and below (right, $T=0.7$ ) the binding transition.

while short-range attraction results from the combination with the attractive $r^{-6}$ term. In total this mimics implicit solvent. In accordance with the literature, we cutoff and shift the Lennard-Jones potential to zero at $r_{c}=2.5 \sigma$. This allows the usage of a domain decomposition. We focus on flexible polymers in this work, which undergo a collapse transition at the theta temperature $T_{\theta}$. The total potential energy is then obtained as the sum of interaction potentials.

If the polymers are grafted, we fix one of their end points at a steric surface covering the $x-y$ plane at $z=0$. No monomer is allowed to cross this geometric constraint, i.e., $z_{i}<0$ is forbidden. No further interactions with the (inert) surface are assumed. The end points are grafted with distance $d=r_{0}$ and are immobilized. If the polymers are not grafted, we enclose them in a cubic box of side length $L$ with steric walls. For an illustration see Fig. 1.

We employ Markov chain Monte Carlo simulations to estimate thermodynamic and structural properties in equilibrium. The phase space is commonly reduced to the conformational phase space (or state space) by omitting the kinetic-energy contributions. In principle, this reduction produces the same canonical expectation values and thus the same canonical transition points. It will, however, be relevant for the (conformational) microcanonical analysis (see Sec. 3.2 [37. The first method we consider is the canonical Metropolis algorithm [38, where proposed updates from a state with potential energy $E_{p}$ to a state with potential energy $E_{p}^{\prime}$ are accepted with the probability $\min \left(1, \exp \left[-\beta\left(E_{p}^{\prime}-E_{p}\right)\right]\right)$, where $\beta=1 / k_{\mathrm{B}} T$ is the inverse temperature (we generally set $k_{\mathrm{B}}=1$ ). A more elaborate multicanonical approach [3940. is to consider a generalized ensemble, replacing the Boltzmann weight by a weight function $W\left(E_{p}\right)$, which is iteratively adapted to yield a flat histogram $H\left(E_{p}\right)$ for the acceptance probability $\min \left(1, W\left(E_{p}^{\prime}\right) / W\left(E_{p}\right)\right)$. In this formulation, the optimal result would correspond to the inverse conformational density of states $W\left(E_{p}\right) \approx \Omega\left(E_{p}\right)^{-1}$. This can be seen if one expresses the partition function in terms of a potential-energy integral, i.e.,

$$
Z_{\beta}=\int d E_{p} \Omega\left(E_{p}\right) e^{-\beta E_{p}} \rightarrow Z_{\text {muca }}=\int d E_{p} \Omega\left(E_{p}\right) W\left(E_{p}\right) .
$$

An estimate of the density of states in turn yields direct information about the conformational microcanonical ensemble, since the microcanonical inverse temperature is defined in terms of the density of states (see below) 41/42]43]44]. Canonical expectation values are obtained by histogram and time-series reweighting [45. We follow the multicanonical approach with a parallel implementation [46. For recent overviews of generalized ensemble methods in the field of polymers see, e.g., Refs. [1147]. 

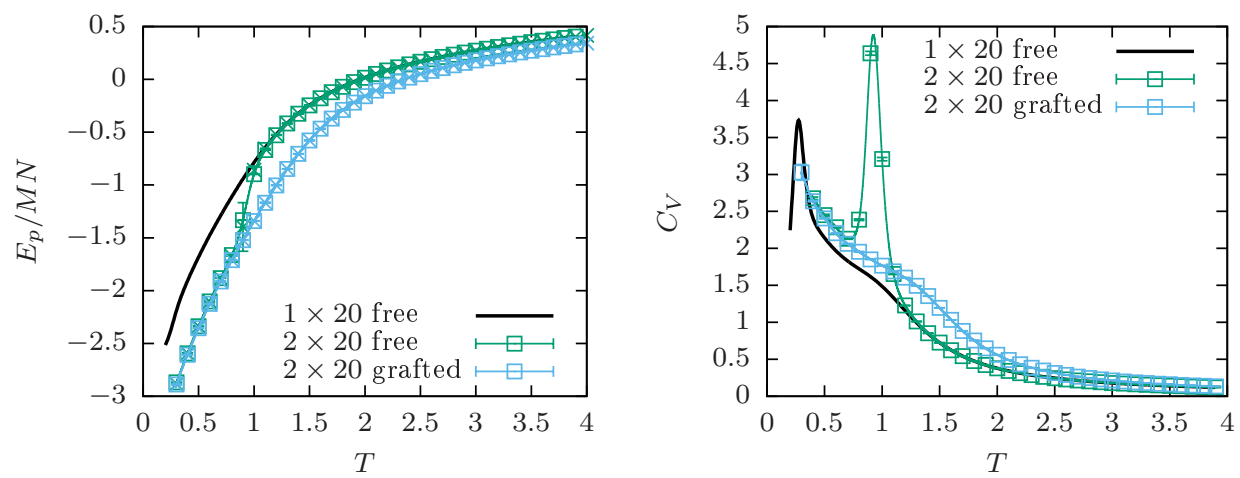

Fig. 2. Estimates of the canonical average potential energy and its derivative, the specific heat, for chains of length $N=20$ from parallel multicanonical (squares and lines) simulations. For the energy we show in addition results from Metropolis simulations (crosses), which are in perfect agreement but suffer precision directly at first-order like transitions. The single-chain behavior is compared to the binding of two chains, both freely moving in a box $\left(\rho=10^{-4}\right)$ and grafted to a steric surface.

Our Monte Carlo updates include short-range single-monomer shifts, long-range polymer displacements (if applicable), and local bond rotations. If for any monomer a shift below the surface $(z<0)$ is proposed, the update is rejected by the steric wall constraint. This generates an effective (entropic) repulsion without any (energetic) potential. Error bars are obtained from statistical fluctuations taking into account the integrated autocorrelation times (for the Metropolis data) and from Jackknife error estimation (for the representative multicanonical data points obtained from timeseries reweighting) [45. From the multicanonical data we additionally estimate highresolution data points by histogram reweighting, which suffers minor discretization effects from binning a continuous energy domain. These form the connecting lines in the presented canonical results below.

\section{Results}

We present our numerical results about the effect of end-point grafting on the binding transition of two polymers from different viewpoints. First, we start with the canonical picture showing the result of grafting on the example of the average energy and average end-to-end distance per polymer. Second, we change to the microcanonical picture, which will allow us to draw qualitative conclusions about the order of the finite-size binding transition.

\subsection{Canonical}

In the first step we will focus on polymer systems with $M$ polymers of length $N=20$. We compare the temperature curves of a single (isolated) free polymer, two polymers at density $\rho=M N / L^{3}=10^{-4}$ in a cubic box with steric walls (i.e., $L \approx 74$ ), and two polymers grafted at a steric surface at distance $d=r_{0}$, the bond length defined in Eq. (1). Figure 2 shows the average potential energy per monomer $E_{p} / M N$ and its derivative, the specific heat $C_{V}=k_{\mathrm{B}} \beta^{2}\left(\left\langle E_{p}^{2}\right\rangle-\left\langle E_{p}\right\rangle^{2}\right) / M N$. The single polymer case $(1 \times 20$ free $)$ shows a high-temperature extended regime signaled by high energies due to low number of contacts. A temperature decrease causes the formation of 


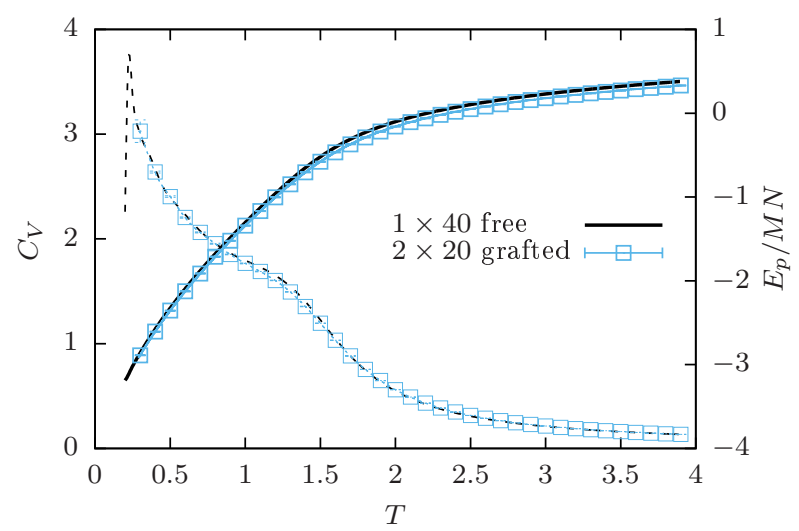

Fig. 3. Comparison of the canonical potential energy $E_{p}$ (solid) and its derivative $C_{V}$ (dashed) for a single polymer of length $N=40$ (black lines) and two grafted polymers of length $N=20$ (blue squares with error bars).

monomer-monomer contacts (compare Fig. 1 (left)), which leads to a decrease in potential energy signaled by a shoulder in the specific heat, see Fig. 2 (right) at $T \approx 1$. This is the continuous collapse transition. The peak in $C_{V}$ upon further temperature decrease corresponds to a freezing transition into more compact states with even lower potential energy. Adding a second polymer at a sufficiently low density (inside a cubic box with steric walls, $2 \times 20$ free) reproduces the same high-temperature behavior because the polymers may be considered isolated. The qualitative behavior changes, initialized by a stronger energy decrease, at the binding (or aggregation) transition. This is also signaled by a pronounced peak in $C_{V}$ at $T=0.925(2)$, where the two polymers bind to each other and entangle to maximize contacts and minimize energy. This is usually argued to be a discontinuous, first-order transition due to the competition of entropy and energy [23|24|25]15]. Upon further temperature decrease the polymer aggregate may be considered to behave almost as a single polymer of length $N=40$ and a further freezing transition may be expected.

If we now graft two end points at the steric surface $(2 \times 20$ grafted), we keep the qualitative low-temperature behavior of two free polymers up to effective repulsive interactions induced by the surface, which are barely noticeable in the average energy. However, since the polymers are fixed to the surface they do not recover the isolated single polymer behavior, having at least two monomers at the surface in close contact. The discontinuous aspects in the binding transition of two free polymers are now altered to features of a continuous binding transition of two grafted polymers: We observe a continuous variation of the average energy and a shoulder in the specific heat. Indeed, the shoulder in the specific heat resembles the signal of a single polymer collapse. Figure 3 shows canonical data for two grafted $N=20$ chains to be very similar to those of a single $N=40$ polymer. This can be understood, considering that the two chains are grafted at a distance $d=r_{0}$, equal to the bond length. Low-temperature conformations are consequently almost indistinguishable, besides the reduction of symmetry by fixing a specific bond to the outer shell of the globule. Even at high temperature the qualitative behavior should be close to that of a single chain with effective repulsive interactions due to a steric surface attached to its middle segment. Thus, a collapse-like binding mechanism is expected, in contrast to the entropy-energy competition for the free counterpart. 

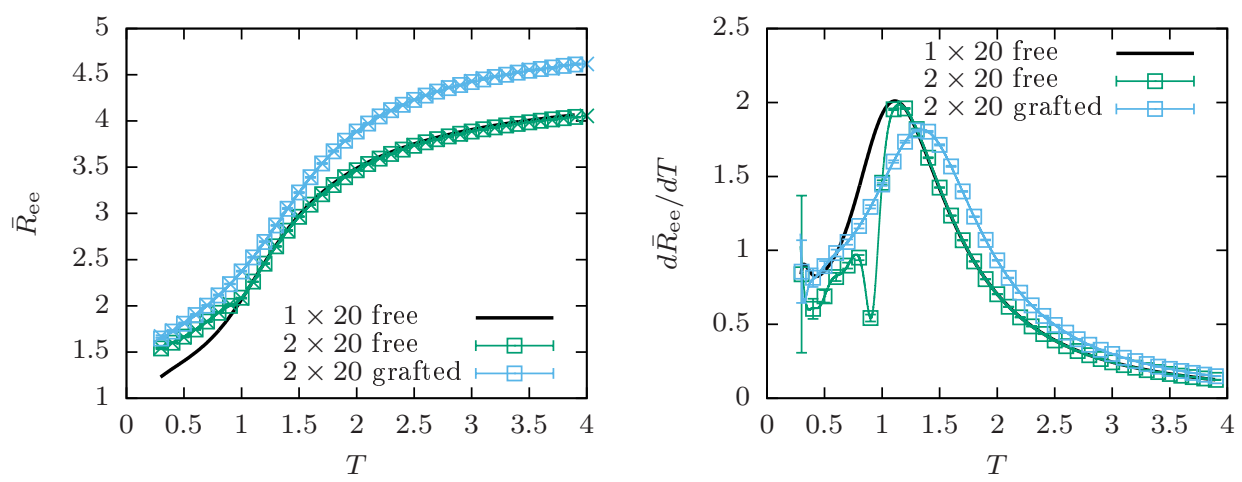

Fig. 4. Estimates of the canonical average end-to-end distance and its derivative for chains of length $N=20$ from parallel multicanonical (squares and lines) simulations. For the endto-end distance we show in addition results from Metropolis simulations (crosses), which are again in perfect agreement. The single-chain behavior is compared to the binding of two chains, both freely moving in a box $\left(\rho=10^{-4}\right)$ and grafted to a steric surface (at distance $\left.r_{0}\right)$.

This observation of a collapse-like process is supplemented in Fig. 4 by looking at the average end-to-end distance, defined as

$$
\bar{R}_{\mathrm{ee}}=\frac{1}{M} \sum_{i=1}^{M} R_{\mathrm{ee}, i},
$$

where $R_{\mathrm{ee}, i}$ is the end-to-end distance of a single polymer, and its thermal derivative $d \bar{R}_{\mathrm{ee}} / d T=k_{\mathrm{B}} \beta^{2}\left(\left\langle\bar{R}_{\mathrm{ee}} E_{p}\right\rangle-\left\langle\bar{R}_{\mathrm{ee}}\right\rangle\left\langle E_{p}\right\rangle\right)$. Again, we present data for a single polymer, two free polymers (confined in a box) and two grafted polymers of length $N=20$. The single-polymer collapse may be observed as a broad peak in $d \bar{R}_{\mathrm{ee}} / d T$ at $T=$ $1.111(6)$. The addition of a second polymer again shows a high-temperature behavior consistent with the single polymer. The small deviation is due to the confinement in a steric box. Simulations with periodic boundary conditions for sufficiently dilute polymers show the identical high-temperature behavior as for isolated chains [24 25]. At a density-dependent temperature within the collapse peak, a sudden decrease to a local minimum signals the binding (or aggregation) transition, here in the regime $T \in(0.9,1.1)$. This is reflected also in the average end-to-end distance by a deviation from the single-polymer behavior. The polymers are thus further extended in the aggregate than in the globule. This signal is not as clear as for larger polymer numbers, where a more drastic rearrangement occurs [24].

When grafting the polymers, we can clearly see the effective repulsive interaction of the steric surface in the average end-to-end distance, cf. Fig. 4 (left). Grafting increases the average end-to-end distance because elongations are only possible away from the surface and thus away from the grafted end point. This is different for the free polymer, where one end point may be in the center of a coil (low temperature) or where an isotropic self-avoiding random walk would cross the surface (high temperature). If one imagines the space of all (symmetric) conformations, then the steric surface cuts off the half space. This removes the symmetric counterparts of the extended chains but removes even more compact chains which form by crossing the surface only with a subset of segments. Thus, the steric surface leads to a stretching of a single chain. In addition, grafting of two chains further analogously stretches the polymers because of the local self-avoidance in the vicinity of the chain ends. The average end-to-end 


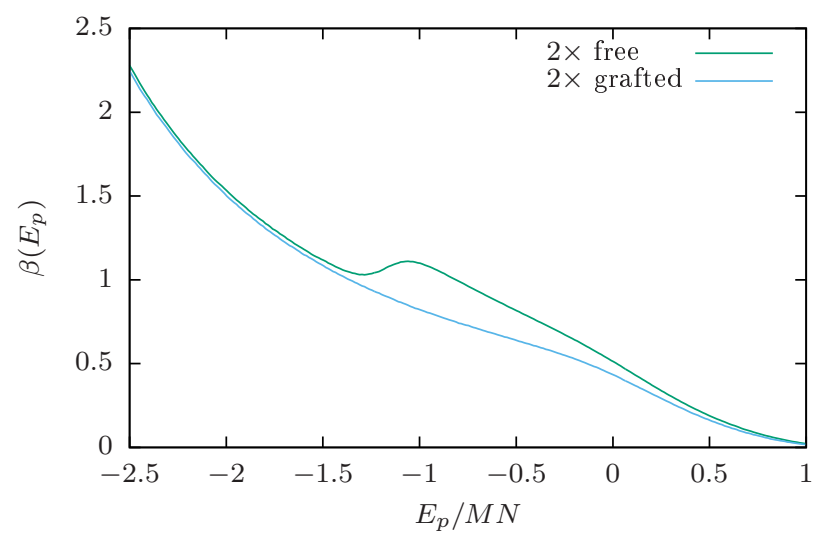

Fig. 5. Estimates of the microcanonical inverse temperature for two chains of length $N=20$ from parallel multicanonical simulations. The free chain shows the expected backbending typical for first-order finite-size transitions. Grafting the chains to a steric surface in close proximity (at distance $r_{0}$ ) diminishes the backbending and results in a second-order finitesize transition.

distance of course shows differences to the single $N=40$ chain, being a local property which cannot be directly related. Instead, one can image that the end-to-end distance of the free ends would show a similar behavior, but this is outside of the present scope. The thermal derivative of the average end-to-end distance shows again a broad transition peak around $T=1.341(5)$. This further supports the coil-to-globule-like character of the structural grafted-binding transition, which was conjectured above.

\subsection{Microcanonical}

We move on to the microcanonical viewpoint 41424344]. Starting from a conformational density of states it is useful to define the conformational microcanonical entropy $S\left(E_{p}\right)=k_{\mathrm{B}} \ln \Omega\left(E_{p}\right)$. The derivative with respect to the potential energy is $d S\left(E_{p}\right) / d E_{p}=k_{\mathrm{B}} \beta\left(E_{p}\right)$ with the microcanonical inverse temperature,

$$
\beta\left(E_{p}\right)=d \ln \Omega\left(E_{p}\right) / d E_{p} .
$$

This quantity encodes finite-size transitions in its inflection points 4144]. The example of two polymers of length $N=20$ is shown in Fig. 5 . For two free polymers at density $\rho=10^{-4}$ we observe a back-bending in $\beta\left(E_{p}\right)$ at $E_{p} / M N \approx-1.2$, which signals a first-order finite-size binding transition. This corresponds to a convex intruder in $S\left(E_{p}\right)$ and can be translated into phase coexistence in terms of a double-peaked canonical probability distribution $P\left(E_{p}\right) \propto \Omega\left(E_{p}\right) e^{-\beta E_{p}}$ 4248] with a latent heat and a free-energy barrier. When grafting the polymers, the back-bending vanishes, while the general behavior for low and high energies remains compatible. We will see below that in the grafted case there is an inflection point with negative slope, which suggests a second-order finite-size binding transition.

The order of the finite-size transition may be well identified in the derivative of the microcanonical inverse temperature,

$$
\gamma\left(E_{p}\right)=d \beta\left(E_{p}\right) / d E_{p}
$$

where the inflection points correspond to peaks. A peak with a positive value refers to a backbending in $\beta\left(E_{p}\right)$ and thus a first-order finite-size transition, while a peak with 


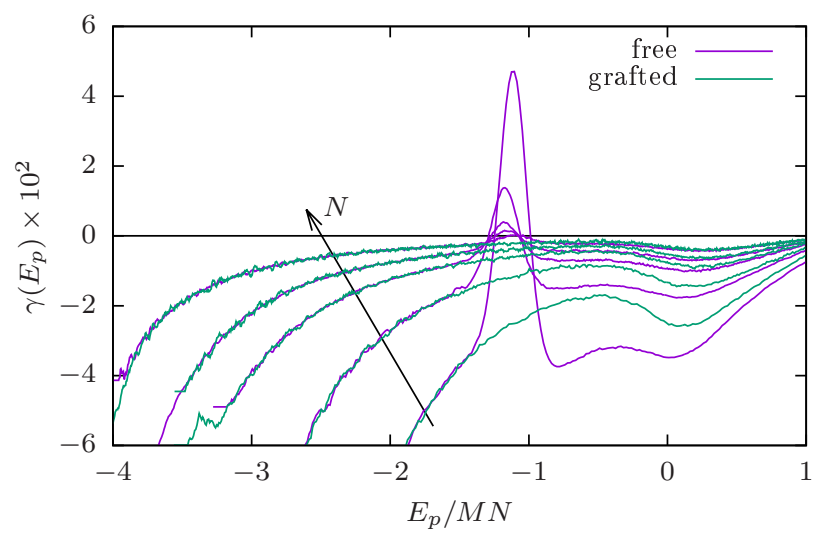

Fig. 6. Microcanonical inflection point analysis of the binding transition of two homopolymer chains for increasing length $N=\{13,20,30,40,60\}$. Compared are two chains in a steric box at fixed density $\rho=10^{-4}$ (free) such that the longest chain is still shorter than the linear box length, and two chains grafted to a steric surface at a distance $r_{0}$ (grafted). The free case shows positive peaks decreasing with polymer length, which signals a finite-size first-order transition possibly with a crossover to a second-order or a tricritical behavior (see discussion in text). The grafted case clearly shows negative peaks increasing with polymer length signaling a second-order finite-size transition, which may be expected to converge to the single-chain collapse in the limit of infinite chain length.

a negative value signals a second-order finite-size transition. In a proper thermodynamic limit, the peaks should approach zero from above and from below, respectively. Figure 6 shows $\gamma\left(E_{p}\right)$ for two polymers of various chain length $N=\{13,20,30,40,60\}$; both either free in a cubic box with steric walls at fixed density $\rho=10^{-4}$, or grafted to a steric surface at distance $d=r_{0}$. We can clearly see that the finite-size binding transition of two free polymers shows a positive peak for all chain lengths around $E_{p} / M N \approx-1$ which rapidly decreases to zero. This peak corresponds to the binding transition, where the small (negative) peak at higher energies may be related to the single-polymer collapse transition.

The binding peak of two free polymers actually seems to reach zero already for finite system sizes. This is an indication that the considered thermodynamic limit is not well defined. In fact, fixing the number of polymers $M$ and the density $\rho=M N / L^{3}$ is not a suitable choice because the single-chain length $N$ will inevitably exceed the linear size of the steric box, since then $N \propto L^{3}$. As a consequence, the effective repulsive interaction induced by the wall increases, leading to more prominent deformations of equilibrium conformations. A more adequate approach towards infinite-size systems is the limit of increasing polymer number $M$ at fixed polymer length $N$ [24 37]. In the present case, we want to focus on the finite-size binding transition of $M=2$ polymers and merely illustrate the transition order for different chain length, with $N<L$ for all cases (for $N=60, L \approx 106$ still).

Upon grafting, the situation changes and we only observe a single negative maximum between $E_{p} / M N \approx-1$ and $E_{p} / M N \approx 0$. For small energies the microcanonical behavior matches with the behavior for the free polymers because the aggregate does not feel a density anymore. The single transition now combines the collapse and binding transition due to the spatial vicinity and the finite-size binding transition may be classified second order. In fact, the systematic approach towards zero suggests that this formulation of increasing chain length of two grafted polymers actually is a valid thermodynamic limit and the binding transition of grafted polymers may be classified 
as a second-order phase transition. We argued above that the binding transition is expected to coincide with the single-polymer collapse for infinite chain length, consistent with our results. General arguments imply also the same mean-field limit of polymer solutions and isolated chains in the limit $N \rightarrow \infty$ (see, e.g., Ref. [49]), which was not accessible in the present scope and is currently under investigation.

\section{Conclusions}

We have shown that grafting two polymers to a steric surface at a close distance results in a second-order binding transition, which is in contrast to the first-order like finite-size binding transition for free polymers in a steric box. This is relevant for an experimental study of polymer binding, where in vitro polymers would be commonly grafted. In this case, one will neither observe a latent heat nor hysteresis effects associated with first-order like transitions, which would be expected to occur in vivo. Still, grafted polymers may be studied with respect to aggregate properties and their dynamics which are expected to sufficiently coincide for observables which are not directly influenced by the geometric constraint. One exception is the average end-to-end distance and subsequently (no longer isotropic) geometric properties.

Interesting effects may be anticipated for interacting surfaces, where the binding of polymers would compete with the surface attraction. Connecting to experimental setups, the surfaces may be considered both flat or curved, e.g., when grafting polymers to nanoparticles.

The project was funded by the Deutsche Forschungsgemeinschaft (DFG) under Grant No. JA 483/31-1. The authors gratefully acknowledge the computing time provided by the John von Neumann Institute for Computing (NIC) on the supercomputer JURECA at Jülich Supercomputing Centre (JSC) under Grant No. HLZ24.

\section{References}

1. J. Schnauß, T. Händler, J. A. Käs, Polymers 8, 274 (2016)

2. D. Strehle, J. Schnauß, C. Heussinger, J. Alvarado, M. Bathe, J. Käs, B. Gentry, Eur. Biophys. J. 40, 93 (2011)

3. F. Huber, D. Strehle, J. Käs, Soft Matter 8, 931 (2012)

4. M. M. A. E. Claessens, M. Bathe, E. Frey, A. R. Bausch, Nat. Mat. 5, 748 (2006)

5. M. Bathe, C. Heussinger, M. M. A. E. Claessens, A. R. Bausch, E. Frey, Biophys. J. 94, $2955(2008)$

6. C. Heussinger, F. Schüller, E. Frey, Phys. Rev. E 81, 021904 (2010)

7. J. Kierfeld, R. Lipowsky, Europhys. Lett. 62, 285 (2003)

8. J. Kierfeld, T. Kühne, R. Lipowsky, Phys. Rev. Lett. 95, 038102 (2005)

9. P. Benetatos, A. von der Heydt, A. Zippelius, New J. Phys. 16, 113037 (2014)

10. J. Kierfeld, Phys. Rev. Lett. 97, 058302 (2006)

11. J. Zierenberg, M. Marenz, W. Janke, Polymers 8, 333 (2016)

12. T. Iwataki, S. Kidoaki, T. Sakaue, K. Yoshikawa, S. S. Abramchuk, J. Chem. Phys. 120, $4004(2004)$

13. M. S. Turner, R. W. Briehl, F. A. Ferrone, R. Josephs, Phys. Rev. Lett. 90, 128103 (2003)

14. G. M. Grason, R. F. Bruinsma, Phys. Rev. Lett. 99, 098101 (2007)

15. J. Zierenberg, W. Janke, Europhys. Lett. 109, 28002 (2015)

16. M. J. Stevens, Phys. Rev. Lett. 82, 101 (1999)

17. M. Rief, F. Oesterhelt, B. Heymann, H. E. Gaub Science 275, 1295 (1997) 
18. M. Tress, E. U. Mapesa, W. Kossack, W. K. Kipnusu, M. Reiche, F. Kremer, Science 341, 1371 (2013)

19. J. N. Bright, D. R. M. Williams, Europhys. Lett. 45, 321 (1999)

20. T. T. Pham, S. K. Pattanayek, G. G. Pereira, J. Chem. Phys. 123, 034904 (2005)

21. P. Benetatos, E. M. Terentjev, A. Zippelius, Phys. Rev. E 88, 042601 (2013)

22. S. Dutta, P. Benetatos, Y. S. Jho, Europhys. Lett. 114, 28001 (2016)

23. C. Junghans, M. Bachmann, W. Janke, Europhys. Lett. 87, 40002 (2009)

24. J. Zierenberg, M. Mueller, P. Schierz, M. Marenz, W. Janke, J. Chem. Phys. 141, 114908 (2014)

25. M. Mueller, J. Zierenberg, M. Marenz, P. Schierz, W. Janke, Physics Procedia 68, 95 (2015)

26. S. Kumar, Y. Singh, Phys. Rev. E, 51, 579 (1995)

27. M. Baiesi, E. Carlon, E. Orlandini, A. L. Stella, Phys. Rev. E, 63, 041801 (2001)

28. P. Leoni, C. Vanderzande, Rev. E, 68, 051904 (2003)

29. A. Ferrantini, E. Carlon, J. Stat. Mech.: Theory and Exp., P02020 (2011)

30. J.-C. Walter, A. Ferrantini, E. Carlon, C. Vanderzande, Phys. Rev. E 85, 031120 (2012)

31. S. Kumar, R. Kumar, W. Janke, Phys. Rev. E 93, 010402(R) (2016)

32. J. Zierenberg, J. Gross, W. Paul, W. Janke, in preparation

33. T. Vogel, J. Gross, M. Bachmann, J. Chem. Phys. 142, 104901 (2015)

34. J. Gross, T. Vogel, M. Bachmann, Phys. Chem. Chem. Phys. 17, 30702 (2015)

35. S. Karalus, W. Janke, M. Bachmann, Phys. Rev. E 84, 031803 (2011)

36. M. Möddel, W. Janke, M. Bachmann, Macromolecules 44, 9013 (2011)

37. J. Zierenberg, P. Schierz, W. Janke, arXiv:1607.08355 (2016)

38. N. Metropolis, A. W. Rosenbluth, M. N. Rosenbluth, A. H. Teller, E. Teller, J. Chem. Phys. 21, 1087 (1953)

39. B. A. Berg, T. Neuhaus, Phys. Lett. B 267, 249 (1991); Phys. Rev. Lett. 68, 9 (1992)

40. W. Janke, Int. J. Mod. Phys. C 03, 1137 (1992); Physica A 254, 164 (1998)

41. D. H. E. Gross, Microcanonical Thermodynamics (World Scientific, Singapore, 2001)

42. W. Janke, Nucl. Phys. B (Proc. Suppl.) 63 A-C, 631 (1998)

43. C. Junghans, M. Bachmann, W. Janke, Phys. Rev. Lett. 97, 218103 (2006)

44. S. Schnabel, D. T. Seaton, D. P. Landau, M. Bachmann, Phys. Rev. E 84, 011127 (2011)

45. W. Janke, Monte Carlo Simulations in Statistical Physics - From Basic Principles to Advanced Applications, in Order, Disorder and Criticality: Advanced Problems of Phase Transition Theory, Vol. 3, edited by Y. Holovatch (World Scientific, Singapore, 2012), p. 93

46. J. Zierenberg, M. Marenz, W. Janke, Comput. Phys. Commun. 184, 1155 (2013); Physics Procedia 53, 55 (2014)

47. W. Janke, W. Paul, Soft Matter 12, 642 (2016)

48. P. Schierz, J. Zierenberg, W. Janke, Phys. Rev. E 94, 021301(R) (2016)

49. W. Paul, T. Strauch, F. Rampf, K. Binder, Phys. Rev. E 75, 06080(R) (2007) 